\title{
A Practical Approach for Magnetic Core-Loss Characterization
}

\author{
F. Dong Tan, Member, IEEE, Jeff L. Vollin, Member, IEEE, and Slobodan M. Cuk
}

\begin{abstract}
A practical approach for magnetic core-loss characterization up to a few megahertz is presented. An error analysis is for the first time performed, revealing that corrections are needed to compensate for errors introduced by the extra phase shift inherent in a measurement setup, and by shunt parasitic capacitance associated with an inductive device under test. $A$ simple technique is then proposed to control the error so as to satisfy prescribed tolerances. Extensive measurements done on a TDK PC40 core yield results which support the analysis. Several sample cores are then characterized at a few megahertz.
\end{abstract}

\section{INTRODUCTION}

I $\mathrm{N}$ magnetic designs, the core loss and the permeability are two essential parameters representing inherent characteristics of a magnetic material [1]. Often a designer may find that such data are not available for either a particular frequency or a particular flux density. In this situation, the designer has to derive approximate values by either extrapolating available data or by experimental measurement techniques. This is particularly true for research and development purposes, since sample-to-sample variations of a given core material can be as high as up to $\pm 30 \%$, according to [2].

Because of the nature of the magnetic field and the nonlinear mechanisms involved in the process of magnetization, the core loss and the permeability cannot be expressed by accurate, yet simple closed-form formulae. Hence, approximate models, together with experimental techniques, are frequently used instead.

There are basically two categories of measurement techniques: indirect and direct methods. The indirect method is typified by the calorimetric method. It enjoys high accuracy in measuring the dissipated power. However, there are inherent disadvantages such as: It is very difficult to set up, and it cannot distinguish the copper loss (or the core loss) from the total loss.

Direct methods measure directly the voltage and current, and then construct the loss power in a certain way. An appealing advantage is that it is easy to set up and to reproduce a measurement. Also, as reported in [3], a direct method generated results which were confirmed by an independent calorimetric method to be within $\pm 4 \%$ in error. Direct

Manuscript received December 7, 1993; revised October 17, 1994. This work was supported by the Missile Systems Group, Hughes Aircraft Co., Canoga Park, CA

F. D. Tun is with QSC Audio Products Inc., Costa Mesa, CA 92626 USA

J. L. Vollin is with Hughes Missile Systems Company, Tucson, AZ 85721 USA.

S. M. Cuk is with the California Insitute of Technology, Pasadena, CA 91125 USA.

IEEE Log Number 9408501 . methods have been popular for simplicity and reasonable accuracies.

Many direct methods have been reported in the literature (see, for example, [4]-[9]). While most of the authors realized that the extra phase shift associated with parasitics of a measurement setup can introduce significant error, no effective methods were proposed to deal with this notorious error. Common practices have been limited either to use awkward methods to control the extra phase shift or to resort to exotic equipment. An effective solution to the problem is proposed here.

It is the purpose of this paper to present a practical, yet accurate approach for characterizing magnetic core materials. This approach is practical since it is easy to use and it employs conventional equipment. It is also accurate since it has an error analysis which can be utilized to satisfy prescribed tolerances. Furthermore, the whole approach is independent of models for the core loss and permeability selected for a particular application.

\section{Outline of Discussion}

Section II discusses selection of models for the core loss and permeability. Section III describes details of the measurement setup. An approximate equivalent circuit is derived for the toroidal two-winding transformer, which facilitates the error analysis. Details on how to obtain data with dc bias are also included. Section IV presents an error analysis for measurements of the core loss as well as the permeability. Useful expressions are derived for determination of the core loss and permeability, satisfying prescribed tolerances. Section $\mathrm{V}$ provides core loss data at several hundred kilohertz for TDK PC40 and compares them to typical data published by TDK. These two sets of data have good agreement, supporting the analytic results. Then core loss and permeability data are obtained for several sample materials at megahertz frequencies. Section VI summarizes main results.

\section{SElection of APPRoXimate MOdels}

In this section, two models are selected to illustrate the proposed approach. Note that the proposed approach is independent of the selection of models.

\section{A. The Core Loss}

The core loss is the sum of three components: the hysteresis loss, the eddy current loss, and the residual loss. The hysteresis loss is due to the multi-valued nature of the hysteresis loop. 
Suppose that a magnetic specimen is excited from zero to the maximum field and then back to zero field. At the end, the returned power is observed to be less than the supplied. The lost power can basically be considered to have been used for the reorientations of the magnetic domains. This loss is proportional to the area encircled by the upper and the lower traces of the hysteresis loop. The area can be obtained by an intergation and is proportional to $B^{2}$. Furthermore, if the shape of the loop remains the same for each succesive recitation, the lost power is simply the product of the area and frequency. Hence, the hysteresis loss is directly proportional to frequency $f$ and the square of peak flux density $B^{2}$ [10], [11].

If a time-varying magnetic field is applied to a specimen, eddy currents will be induced. These currents generate a certain amount of ohmic loss, which is normally called the eddy current loss. To reduce eddy current loss, materials with high resistivities such as ferrites are preferred. However, high resistivities are usually coupled with low permeability. Hence, a tradeoff exists between them for material designs. The mechanism for eddy current loss is the same as that in an conductor. Therefore, the eddy current loss is proportional to $f^{2}$ and $B^{2}$ [12].

The hysteresis loss and the eddy current loss account for a large portion of the total core loss. The rest of it is normally called the residual loss. Mechanisms behind these are complex. Experiences indicate that the residual loss is proportional to frequency $f$ and peak flux density $B$.

Since all three components are, to variable extent, related to $f$ and $B$, the following empirical formula, which is frequently used in industry [13], is chosen as the model for the core loss,

$$
P_{f e}=k V_{e} f^{m} B^{n},
$$

where $V_{e}$ is the volume of the core, $f$ the operating frequency, and $k, m$, and $n$ are constants.

\section{B. The Permeability}

Permeability represents the ability of a given material to be magnetized under various conditions. The characteristic of magnetization is highly nonlinear, which directly translates into permeability's strong dependence on flux density. Different applications have different flux densities, and may call for different models. For example, for Ni-Zn ferrites, under low flux densities, the following model was found to fit the measured data very well [7],

$$
\mu=\mu_{0} \mu_{r} \sqrt{1+\alpha^{2} H^{2}}
$$

\section{Curve Fitting}

The mathematical models for the core loss and the permeability require the determination of the constants $\mu_{r}, \alpha, k, m$, and $n$. These constants can be obtained by the technique of curve-fitting. The returned parameters, $\mu^{*}, \alpha^{*}, k^{*}, m^{*}$, and $n^{*}$, can then be regarded as inherent material constants. Since the technique of curve fitting is well-known, details of it are not pursued further.

In the following, (1) and (2) will be used to illustrate the steps of determining the core loss and permeability. In practice,

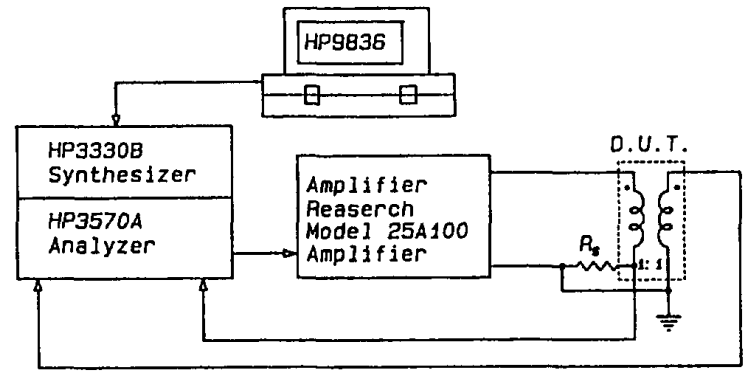

Fig. 1. Schematic of the measurement setup.

one can use models which best fit a particular application. The selected models may very well be different from those used here, but the approach is still applicable.

\section{II. Measurement Setup}

Magnetic core manufacturers usually provide the loss data for symmetrical sinusoidal flux densities, because of its convenience for measurements and specifications. This practice is followed here.

Also, sinusoidal data can be used to estimate data for nonsinusoidal cases [13], [14]. For example, it was found recently in [14] that the core loss corresponding to a bipolar square-wave voltage is related to that of a sinusoidal voltage by a multiplying factor $8 / \pi^{2}$.

Fig. 1 is a general schematic illustration of the actual measurement setup. An HP3330B synthesizer and an HP3570A network analyzer are used for the acquisition of measured data. A sinusoidal voltage from the synthesizer is amplified by an Amplifier Research Model 25A100 amplifier. This voltage is then applied across the primary of a toroidal transformer. The secondary winding is wound in a bifilar fashion with a turns-ratio 1:1. The measured voltage is the one across the open-circuited secondary terminals. The measured current is the sensed current from the current sensing resistor. This configuration can avoid the effect of the primary leakage impedance, i.e., the leakage inductance and the winding resistance, to the extent that the equivalent lumped circuit model is valid [15], [16].

The flux density is directly monitored by a scope, viewing the waveform of the measured voltage, since this voltage is proportional to the derivative of the flux density with respect to time. During all measurements this waveform is closely monitored for any possible distortion in order to guarantee the desired sinusoidal flux density. For high frequency applications (above a few hundred kilohertz), most designs are core loss limited. The flux density is kept well below saturation and the device works primarily on the linear part of the B-H loop. Therefore, the distortion can be assumed negligible.

Fig. 2 is a schematic diagram of the measurement fixture which is built with special attention to minimize parasitic capacitance and inductance. The sensing resistor is implemented with 10 carbon resistors connected in parallel. Its frequency response was measured to be flat from $1 \mathrm{kHz}$ to $20 \mathrm{MHz}$. A $70 \mu \mathrm{H}$ inductor is introduced to bypass the de current in case a dc bias test is performed. A dc bias current $I_{d c}$ is 


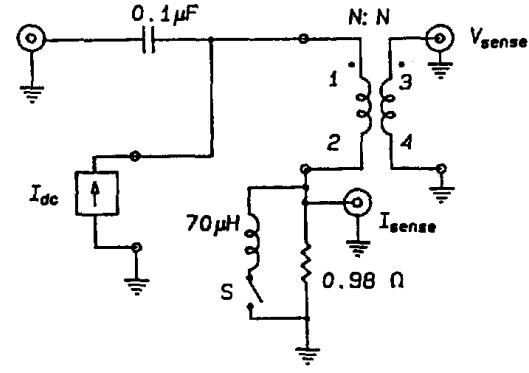

Fig. 2. Schematic for the measurement fixture.

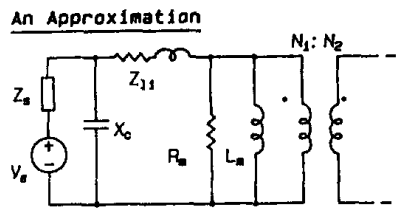

(a)

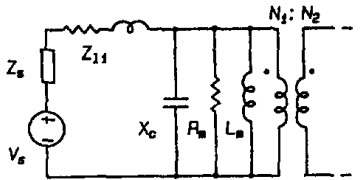

(b)
Fig. 3. Equivalent circuits for a two-winding transformer.

generated from an HP6130C digital voltage source controlled by an HP9836 computer. The fixture with a dc bypass inductor was checked for its frequency response. No additional phase shift is introduced in the frequency range of interest.

The whole measurement setup is calibrated with terminals 1,2 , and 3 all shorted together. In this way all remaining parasitics associated with coax cables, probes, etc., are all accounted for.

Each sample core is demagnetized before a measurement by reducing the magnitude of the ac excitation voltage gradually to zero (dynamic demagnetization).

\section{An Equivalent Circuit}

Fig. 3(a) is a general equivalent circuit model for a transformer. It was used successfully for very wide-band RF transformer designs in the frequency range from $100 \mathrm{kHz}$ to $30 \mathrm{MHz}$ [16]. $Z_{s}$ is the output impedance of the source. $X_{c}$ is reactance presented by the shunt parasitic capacitance of an inductive winding. Note that it also includes the input capacitance of a voltage probe used to measure the voltage. $Z_{l 1}$ is the series combination of the leakage inductance and the winding $\mathrm{dc}$ and ac resistances. $L_{m}$ is the magnetizing inductance associated with the main flux of the transformer. $R_{m}$ is a series resistance representing the core loss.

For the purpose of determine a permeability with sufficient accuracy, an approximate circuit of Fig. 3(b) is preferred. The condition for a good approximation can be established as;

$$
Z_{s}+Z_{l 1} \ll X_{c},
$$

which is normally well satisfied since the parasitic capacitance is on the order of ten picofarads.

\section{ERROR ANALYSIS}

Error associated with core loss measurement is first analyzed. Then the effect of parasitic capacitance is examined.

\section{A. Correction for Phase Angle}

The loss power $P$ of the device under test can be constructed from the measured voltage $V$, current $I$, and the phase angle $\phi$ by

$$
P=V I \cos \phi .
$$

The accuracy of the constructed power is largely dependent on the accuracy of the measurement for the phase angle $\phi$, which is discussed further in the following. The total increment of the power is given by

$$
\Delta P=\frac{\partial P}{\partial V} \Delta V+\frac{\partial P}{\partial I} \Delta I+\frac{\partial P}{\partial \phi} \Delta \phi .
$$

Since the voltage and the current can usually be measured with sufficient accuracy, $\Delta V$ and $\Delta I$ can be assumed to be diminishingly small. Hence the total increment of the power can be rewritten as

$$
\Delta P=\frac{\partial P}{\partial \phi} \Delta \phi
$$

where $\Delta \phi$ is the phase error from the measurement process, and $\Delta P$ the power error due to $\Delta \phi$.

Straightforward algebra leads to the following expression for the relative power error:

$$
\left|\frac{\Delta P}{P}\right|=|-\tan \phi \Delta \phi|=|\tan \phi||\Delta \phi| .
$$

Rich information can now be extracted from (7). First, it captures explicitly and elegantly, for the first time, the problem of high error sensitivity of the constructed power $(\Delta P / P)$ over the phase error $(\Delta \phi)$. It is seen that as $\phi$ approaches $90^{\circ}, \Delta \phi$ has to go to zero in order for $\Delta P / P$ to have a finite value. Since it is virtually impossible to eliminate $\Delta \phi$ in an actual measurement setup, any effort to control $\Delta \phi$ is doomed to failure. This is an essential difficulty which plaques the direct method (by limiting the accuracy).

Second, careful examination of (7) reveals that, while exposing the problem, it also provides a simple, yet effective solution. Indeed, (7) indicates that for a certain value of $\Delta \phi$, the value of $\tan \phi$ can be controlled, in order for $\triangle P / P$ to have a diminishingly small value.

In other words, engineering efforts focused on the control of the value for $\phi$, instead of $\Delta \phi$, would be easier and much more effective. For example, take the relative power error to be $10 \%$, then straightforward algebra yields,

$$
-\frac{1}{10|\Delta \phi|} \leq \tan \phi \leq \frac{1}{10|\Delta \phi|} \text {. }
$$

For any given phase error $|\Delta \phi|$, the above inequality gives a feasible region of the phase angle $\phi$ within which the measured power will be at most $\pm 10 \%$ away from the actual value. For example, if the phase shift is limited to be $|\Delta \phi|=3^{\circ}$, the corresponding feasible region of the phase angle is given by $|\phi| \leq 63^{\circ}$.

In an actual measurement, the phase angle may not be in the feasible region. One typical example is a measurement of a core material with low core loss. For a finite value of inductance, very low loss (a vanishingly small value for the 


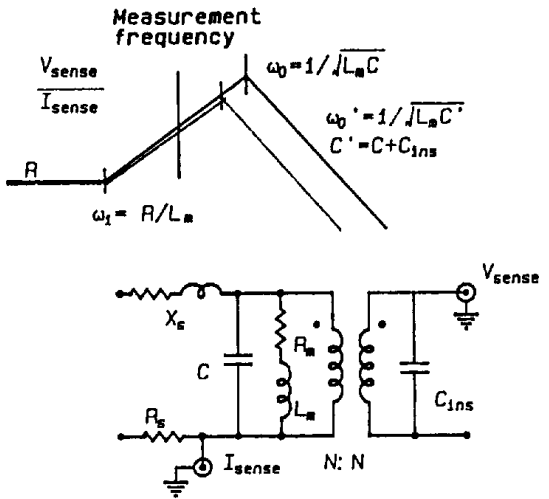

Fig. 4. Bring the phase angle to the desired value.
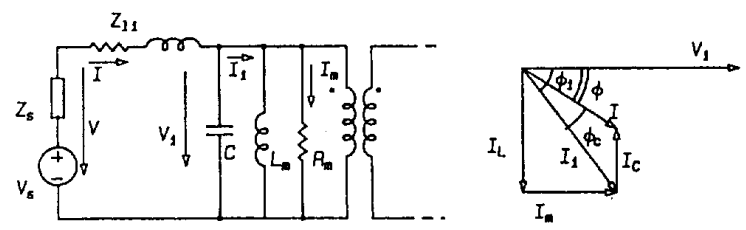

Fig. 5. A correction for measured current.

resistance) directly translates into a phase angle close to $90^{\circ}$, where error sensitivity is very high. Under this circumstance, a low loss capacitor can be connected across the secondary (or the primary) to bring the phase angle to the desired value (Fig. 4 illustrates the underlying principle).

This technique was mentioned in [17], [18], but its importance was not fully realized.

Note that introduction of an additional capacitor will degrade the accuracy. There are two things one needs to look out for. Additional capacitance in the primary may upset the condition for the equivalent in Fig. 3(b). Additional capacitance in the secondary may worsen the loading on the secondary which exists due to the small input capacitance of the probe.

However, the degradation can be compensated for by tightening up the prescribed tolerance from, say, $10 \%$ to $5 \%$. Equation (7) then gives that the feasible region is reduced from $\pm 63^{\circ}$ to $\pm 45^{\circ}$. Note that this self-adaptive feature of the approach, although advantageous, cannot eliminate the error due to the added capacitance.

It is important to point out that, compared to the error for the case with no added capacitance, the error for the case with added capacitance is not only much less (an order of magnitude reduction) but also well-controlled.

\section{B. Correction for Measured Current}

The parasitic capacitance associated with the toroidal transformer can also introduce error in determining the permeability. Fig. 5 shows a derived approximate equivalent circuit together with the corresponding phasor diagram of various currents. $V_{1}$ is the measured voltage, $I$ is the measured current, and $I_{1}$ is the current needed for determination of the permeability. $L_{m}^{\prime}$ and $R_{m}^{\prime}$ are the corresponding parallel impedance equivalent to the series impedance consisted of $L_{m}$ and $R_{m}$. Since $I_{1}$ cannot be measured physically, it will have to be derived from other measurable quantities. From the principle of the conservation of energy, the following should be met,

$$
P=V_{1} I \cos \phi=V_{1} I_{1} \cos \phi_{1}
$$

which leads to

$$
I_{1}=\frac{\cos \phi}{\cos \phi_{1}} I
$$

where $\phi_{1}=\phi+\phi_{c}$. The angle $\phi_{c}$ is due to the capacitive current $I_{c}$, and its value can be determined from an independent measurement of the frequency response of the impedance defined by $V_{1} / I$.

It can be seen that the closer the measuring frequency is to the self-resonance frequency of the inductive device under test, the greater the error is due to $I_{c}$.

\section{The Proposed Procedure}

Based on results so far obtained, the following procedure can be formulated for practical determination of the core loss as well as the permeability:

- Select (or derive) proper models for a particular application.

- Measure the impedance $\left(V_{1} / I\right)$ of the toroidal transformer to be tested, and determine $\phi_{c}$.

- Use $I_{1}=\left(\cos \phi / \cos \phi_{1}\right) I$ to generate data for permeability fit.

- Determine the overall phase error of the measuring setup $\Delta \phi$, and choose the tolerance for core loss fit.

- Determine the feasible region for the phase angle, according to (7).

- Check if the actual angle is in the feasible region or not. If not, use a low-loss capacitor to bring the angle to the desired value.

- Generate data for core loss fit.

- Perform the curve fitting, respectively.

\section{MEASURED Results}

In this section actual measurement data is presented to support the amalytical results.

\section{A. Core Losses}

A TDK T16-28-13 core was used for test purposes. Data were generated according to the proposed procedure at 50 , $100,200,300,400$, and $500 \mathrm{kHz}$, respectively. With each frequency, the flux density was swept. Flux densities were maintained in the same range, $50-320 \mathrm{mT}$, for all measurements. Table I is a summary of the determined $k^{\prime} s, m^{\prime} s$, and $n^{\prime} s$ from the curve fitting.

The core loss for TDK PC40 material can be represented by

$$
P_{f \mathrm{e}}=2.08 \times 10^{-6} f^{1.43} B^{2.41}
$$

where $P_{f e}$ is in $W / \mathrm{cm}^{3}, f$ in $\mathrm{Hz}$, and $B$ peak value in Tesla. 
TABLE I

Determined Parameters for CORE LOSSES fOR TDK PC40

\begin{tabular}{|c|c|c|c|c|}
\hline \hline Freq.(kHz) & $k^{*}$ & $m^{*}$ & $n^{*}$ & $F^{*}$ \\
\hline 50 & 2.10 & 1.43 & 2.41 & 0.119 \\
\hline 100 & 2.09 & 1.42 & 2.41 & 0.065 \\
\hline 200 & 2.02 & 1.42 & 2.38 & 0.038 \\
\hline 300 & 2.09 & 1.43 & 2.40 & 0.040 \\
\hline 400 & 2.10 & 1.43 & 2.43 & 0.062 \\
\hline 500 & 2.10 & 1.44 & 2.42 & 0.074 \\
\hline Average & 2.08 & 1.43 & 2.41 & 0.066 \\
\hline
\end{tabular}

The data provided by TDK gives

$$
P=2.0 \times 10^{-6} f^{1.46} B^{2.57} \text {. }
$$

The small difference between the two may be attributed to two facts: 1) the sample-to-sample variation of a core material; and 2) the anisotropy compensation temperature for $\mathrm{PC} 40$ is placed around $80^{\circ}$ according to TDK data. Our measurements were, however, done at room temperature. Hence the loss is expected to be slightly larger. Therefore, the agreement of the two sets of data is good, validating the proposed approach.

The proposed approach is then applied to several sample cores. They are TDK's PC40, and K6A, and Ceramics Magnetics' CMD5005, CN20, MN8CX, and C2025. PC40 and $\mathrm{MN} 8 \mathrm{CX}$ are $\mathrm{Mn}-\mathrm{Zn}$ ferrites, and the rest of the cores are $\mathrm{Ni}-\mathrm{Zn}$ ferrites. Since data for most magnetic materials at a few hundred kilohertz are available from manufacturers, the characterizations of the sample materials were conducted at frequencies around $1 \mathrm{MHz}$ where data is not readily available.

Table II summarizes the determined parameters for core losses of sample cores at $1 \mathrm{MHz}$. The determined values for $m$ and $n$ are both close to two. It is therefore seen that under our measurement conditions, the eddy current loss dominates.

Fig. 6 presents core losses for $\mathrm{PC} 40$ at different dc bias current values. It can be seen that the core loss increases drastically with the increase of dc bias current. The implication of this result is significant. A magnetic device with a dc bias flux can have core loss several times larger than what is expected from using manufacturer's data with no dc bias.

It is pointed out that the resources and timing didn't allow an investigation into the physical explanation of the large increase of core loss to proceed at the time this project was performed.

\section{B. Permeabilities}

Table III summarizes the determined $\mu^{\prime}$ s and $\alpha^{\prime}$ s for $\mathrm{Ni}-\mathrm{Zn}$ cores at $1 \mathrm{MHz}$, where (2) was used.

Permeability variation data as a function of dc bias field are valuable for designing magnetic devices with controllable reluctances. A magnetic regulator characterized in [19] is such a device. The proposed approach can handle cases with dc bias without any further difficulties. Fig. 7 shows the variations of the permeability with changes of dc bias current. Permeabilities are observed to be decreasing with the increase of $\mathrm{dc}$ bias current, exhibiting a saturation pattern.
TABLE II

Determined Parameters for CORE Losses at $1 \mathrm{MHz}$.

\begin{tabular}{|c|c|c|c|c|}
\hline \multirow{2}{*}{$\begin{array}{c}\text { Core Materials } \\
\text { at 1MHz }\end{array}$} & \multicolumn{4}{|c|}{ Determined Parameters } \\
\cline { 2 - 5 } & $k^{*}$ & $m^{*}$ & $n^{*}$ & $F^{*}$ \\
\hline CMD5005 & 2.04 & 1.96 & 2.03 & 0.419 \\
\hline CN20 & 2.49 & 1.97 & 2.06 & 0.752 \\
\hline MN8CX & 2.43 & 1.92 & 2.08 & 0.525 \\
\hline C2025 & 2.04 & 2.07 & 1.99 & 0.137 \\
\hline PC40 & 2.02 & 1.90 & 2.03 & 0.047 \\
\hline K6A & 1.97 & 2.03 & 2.00 & 2.056 \\
\hline
\end{tabular}

TABLE III

Determined Parameters for Permeabilities at lMHz.

\begin{tabular}{|c|c|c|c|}
\hline \multirow{2}{*}{$\begin{array}{c}\text { Core Materials } \\
\text { at } 1 \mathrm{MHz}\end{array}$} & \multicolumn{3}{|c|}{ Determined Parameters } \\
\cline { 2 - 4 } & $\mu_{r}^{*}$ & $\alpha^{*}$ & $F^{*}$ \\
\hline CMD5005 & 144.0 & 0.09 & 0.795 \\
\hline CN20 & 84.6 & 0.09 & 3.160 \\
\hline
\end{tabular}

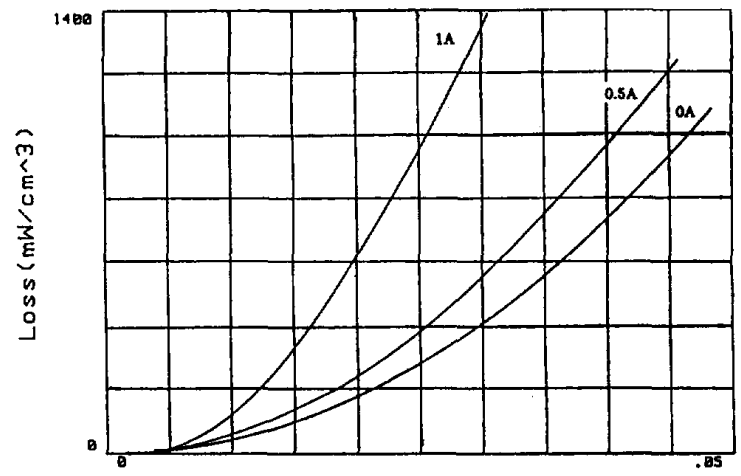

Flux density (T)

Fig. 6. Core losses for PC40 with dc biases.

It should be noted that with dc bias, the permeability actually measured is the incremental permeability. This permeability should be distinguished from the differential permeability [11].

Finally, it is pointed out that the sample cores were characterized with a fixed frequency while sweeping the flux density. The proposed approach will have no difficulty to accommodate frequency sweeping. It ought to be mentioned that if a program code is written for simultaneous frequency and flux density sweeping, curve-fitting can be expected to produce more elegant results.

\section{CONCLUSIONS}

A practical approach for characterizing magnetic materials is proposed. This approach is accurate and yet simple to use. It uses conventional equipment and can be tailored to satisfy prescribed tolerances. 


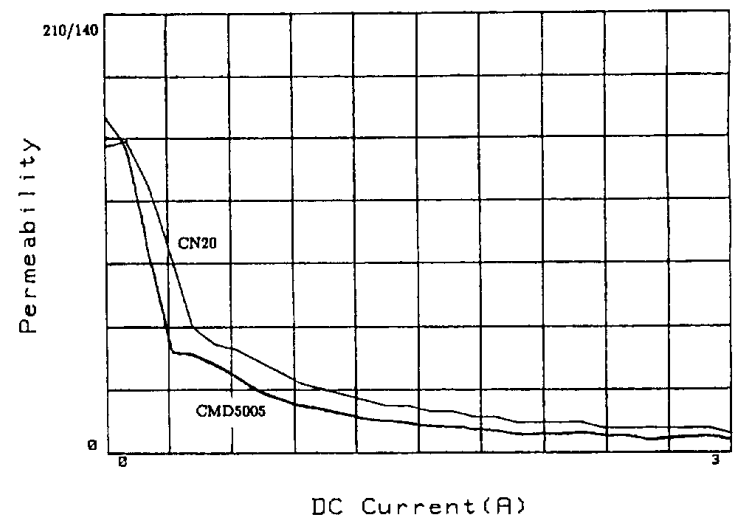

Fig. 7. Permeabilities with de biases.

An error analysis is performed for the first time. A technique is then proposed to control the notorious error sensitivity due to the extra phase shift. Also, a correction is found to be necessary for the determination of permeability to compensate for the effect of the parasitic shunt capacitance associated with an inductive device under test.

A TDK PC40 sample core is measured extensively at $50-500 \mathrm{kHz}$. Results are compared with typical data supplied by TDK. Good agreement is observed, validating the proposed approach.

This approach can be used to determine core losses as well as permeabilities for a given magnetic material at different frequencies and flux densities, in the event that the data are not available or the sample-to-sample variation has to be considered. Applications of the approach are independent of the selection of models for the core loss and permeability.

This approach is equally applicable to cases where de bias is present. The core loss data for $\mathrm{PC} 40$ reveal that the actual core loss with de bias can be several times larger than what manufacturer"s data with no de bias suggest. Permeability data with de bias are also obtained.

\section{ACKNOWLEDGMENT}

The assistance is gratefully acknowledged of Fen Chen, a former member of the Power Electronics Group from 1990 to 1991, for the help in writing program codes in HP Basic for curve-fitting.

\section{REFERENCES}

III S. M. Cuk. "Basics of swikhed-mode power conversion: Topologies, magnetics, and control," Adrances in Switched-Mode Pover Conversion. Vol. II. Teslaco, 1983, pp. 292-305.

121 T. Mitsui and G. V. Schaick, "Ferrite power material for high-frequency upplications." Powertechnics Magazine, pp. 15-19, Feb. 1991.

[3] D. K. Conroy, G. F. Pierce. and P. R. Troyk, "Measurement techniques or the design of high frequency SMPS transformers," IEEE APEC Prot:, 1988, pp. 341-351.

I4I B. Carsten. "Fast, accurate measurement of core loss at high frequencics." PCIM Proc., 1986, pp. 14-24.

151 T. Sato and Y. Sakaki, "100kHz-10MHz iron loss measuring system," IEEE Trans. Magn., vol. 23, pp. 2593-2595, Sep. 1987.

|6| V. J. Thotuvelil, T. G. Wilson, and H. A. Owen, "High-frequency measurement techniques for magnetic cores," IEEE Trans. Pow'er Electron. vol. 5, pp. 41-53, Jan. 1990 .
[7] A. F. Goldberg, "High field properties of Nickel-Zinc ferrites at 1-10MHz,"' IEEE APEC Proc., 1988, pp. 311-318.

[8] P. M. Gradzki and F. C. Lee, "High frequency core loss characterization technique based on impedance measurement," HFPC Proc., 1991, pp. $108-115$.

[9] J. A. Ferreira and J. van Wyk, "Experimental evaluation of losses in magnetic components for power converters," IEEE Trans. Ind. Applicat. vol. 27, pp. 335-339, 1991.

[10] J. K. Watson, Applicat. Magnetism. New York: John Wiley, 1980.

[11] M. R. Bozorth, Ferromagnetism. New York: D. Van Nostrand, 1951.

[12] E. C. Snelling, Soft Ferrites: Properties and Applications, Second Ed. England: Butterworths, 1988.

[13] R. Severns, "HF Core Losses for Non-sinusoidal Waveforms," HFPC Proceedings, 1991, pp. 140-148.

[14] W. Roshen, "Ferrite core loss for power components design," IEEE Trans. Magn., vol. 27, pp. 4407-4415, Nov. 1991.

[15] H. P. J. Wijn and J. J. Went, "The magnetization process in ferrites," Physica, vol. XVII, no. 11-12, pp. 976-992, 1951.

[16] D. Maurice and R. H. Minns, "Very-wide band radio-frequency transformers," Wireless Engineering, June 1947, pp. 168-177, and July 1947 , pp. 209-216.

[17] D. Y. Chen, "Comparisons of high frequency magnetic core losses under two different driving conditions: $A$ sinusoidal voltage and a square-wave voltage," IEEE PESC Record, 1978, pp. 237-241.

[18] D. Y. Chen, "High-frequency core loss characteristics of amorphous magnetic alloy," Proc. IEEE, vol. 69. July, 1981, pp. 853-855.

(19) J. L. Vollin, F. D. Tan, and S. M. Ćuk, "Magnetic regulator modeling," IEEE APEC Proc, 1993, pp. 604-611.

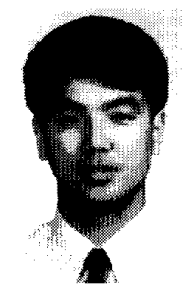

F. Dong Tan earned the B.E.Sc. degree from the Jiangxi Polytechnic University, Nanchang, China, in 1979 and the $\mathrm{Ph} . \mathrm{D}$ degree from the California Institute of Technology, Pasadena, CA, in 1993 , both in electrical engineering.

From 1979 to 1986 , he was with the Faculty of Jiangxi Polytechnic University, where he taught several courses in circuit, electronics, and electric machines. From 1986 to 1987, he was a Visiting Research Assistant Professor at the University of Waterloo, Ontario, Canada, where he applied bifurcation theory to switching converters. From 1987 to 1988, he pursued graduate studies at the University of Victoria, British Columbia. Canada, where he worked on quasi-resonant converters. In 1989, he was accepted directly into the Ph.D. program with the Power Electronics Group at Caltech, where he focused his research on modeling and control of dc/dc converters and associated magnetic design issues. Since November 1993, he has been a Senior Engineer with QSC Audio Products Ine., Costa Mesa, CA. developing softswitched, high performance, and high power professional audio amplifiers. He has published more than 10 peer-reviewed journal and conference papers. He is also one of the inventors of 6 U.S. and European patent applications.

Dr. Tan received several awards for teaching excellence, presented by Jiangxi Polytechnic University.

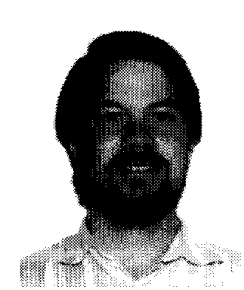

Jeff L. Vollin (S'89-M'92-S'92-M'93) was born in Billings, MT, in 1960. He received the B.S. and Ph.D degrees from the California Institute of Technology, Pasadena, CA, in 1982 and 1993, respectively. He received the M.S. degree from UCLA, Los Angeles, CA, in 1984.

He was a member of the Caltech Power Electronics Group from 1988 to 1993 where he specialized in the modeling of high frequency resonant power converters with applications in high voltage and the modeling of variable-inductance magnetic struc tures. Since 1982, he has been with Hughes Missile Systems Company, Tueson, AZ, where he specializes in high voltage power supplies and traveling-wave tube transmitters for radar applications.

Dr. Vollin was the recipient of a Hughes Doctoral Fellowship in Electrical Engineering. 


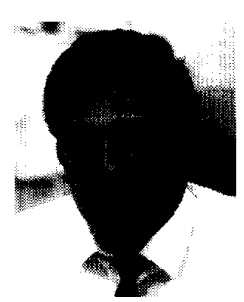

Slobodan M. Ćuk received the B.S.E.E. degree from Belgrade University in 1970, the M.S.E.E. degree from the University of Santa Clara in 1974, and the Ph.D. degree from the California Institute of Technology in 1976.

He is now Professor of Electrical Engineering at Caltech. In 1980, he founded TESLAco with the charter to apply basic research results developed at Caltech to practical commercial amd military designs. He conducts research in electronic energy processing systems and teaches courses in power electronics and the fundamentals of energy processing. In the last 18 years, 25 students obtained Ph.D. degrees in the Power Electronics Group. His publications include over 100 scientific papers in the power electronics field and a three-volume book on switched-mode power conversion. He has taught a number of public and in-house courses around the world, attended by more than 4,000 power electronics specialists. $\mathrm{He}$ is also the major force behind the TESLAco recent developments of high efficiency, high power sensity converter modules and multiple output converters (MOPS). He holds four patents and has over 10 patents pending.

Dr. Cuk won the IR*100 Award in 1980 from Industrial Research magazine for his invention of the Cukonverter ${ }^{\mathrm{TM}}$ and its extensions. He also received the 1991 Edward Longstreth Medal from the Franklin Institute for the invention of integrated magnetics and development of switched-mode power converters, which was the first award to researchers in power electronics in the award's 100-year history. 